\title{
Identifying genes with tri-modal association with survival and tumor grade in cancer patients
}

\author{
Minzhe Zhang ${ }^{1}$, Tao Wang ${ }^{1,2,3}$, Rosa Sirianni ${ }^{4}$, Philip W. Shaul ${ }^{4}$ and Yang Xie , $^{1,25^{*}}$
}

\begin{abstract}
Background: Previous cancer genomics studies focused on searching for novel oncogenes and tumor suppressor genes whose abundance is positively or negatively correlated with end-point observation, such as survival or tumor grade. This approach may potentially miss some truly functional genes if both its low and high modes have associations with end-point observation. Such genes act as both oncogenes and tumor suppressor genes, a scenario that is unlikely but theoretically possible.

Results: We invented an Expectation-Maximization (EM) algorithm to divide patients into low-, middle- and highexpressing groups according to the expression level of a certain gene in both tumor and normal patients. We found one gene, ORMDL3, whose low and high modes were both associated with worse survival and higher tumor grade in breast cancer patients in multiple patient cohorts. We speculate that its tumor suppressor gene role may be real, while its high expression correlating with worse end-point outcome is probably due to the passenger event of the nearby ERBB2's amplification.
\end{abstract}

Conclusions: The proposed EM algorithm can effectively detect genes having tri-modal distributed expression in patient groups compared to normal genes, thus rendering a new perspective on dissecting the association between genomic features and end-point observations. Our analysis of breast cancer datasets suggest that the gene ORMDL3 may have an unexploited tumor suppressive function.

Keywords: Expectation maximization, Oncogene, Tumor suppressor gene, Survival, Breast Cancer

\section{Background}

Alterations in oncogenes or tumor suppressor genes underlie the driving forces of carcinogenesis. An oncogene is a gene that causes cancer through activating mutation or expression at high levels, while for a tumor suppressor gene, it is the loss or reduction of function that leads to cancer. Research in cancer biology has identified hundreds of genes involved in different stages of tumorigenesis [7, 17]. The alterations in these oncogenes or tumor suppressor genes can come from a variety of sources, such as single nucleotide polymorphisms

\footnotetext{
* Correspondence: Yang.Xie@UTSouthwestern.edu

'Department of Clinical Sciences, Quantitative Biomedical Research Center,

University of Texas Southwestern Medical Center, 5323 Harry Hines Blvd, Dallas, TX 75390, USA

${ }^{2}$ Harold C. Simmons Comprehensive Cancer Center, University of Texas

Southwestern Medical Center, 5323 Harry Hines Blvd, Dallas, TX 75390, USA

Full list of author information is available at the end of the article
}

(SNPs), copy number variations (CNV), chromosomal regions, viral integration, gene fusions, etc. There is another type of event called a passenger mutation, which also commonly occurs in tumor tissues. However, such passenger mutations have no effect on the growth of tumors and they usually hitchhike on a near-by tumor driver gene's alteration. It is an important research question to distinguish true tumor driver mutations from artefact events such as passenger mutations in order to better elucidate tumor oncogenesis and evolution. As the names "oncogene" and "tumor suppressor gene" suggest, previous systematic searches for tumor driver genes have mostly adopted the paradigm that a positive association between up-regulation and gain of function vs. tumor proliferation and worse survival hints at a possible oncogene, while for tumor suppressor genes, a negative association is expected. For example, Bric et al. conducted an RNA interference (RNAi) screen for 
tumor suppressors through selecting for small hairpin RNAs (shRNAs) capable of accelerating lymphomagenesis in a mouse model [4]. Koso et al. mobilized the Sleeping Beauty transposon system in mice and profiled insertions that promoted medulloblastoma formation in the cerebellum [15]. Wrzeszczynski et al. carried out a bioinformatics screen for candidate ovarian cancer oncogenes or tumor suppressors by first looking for genes with significant amplification or deletion across tumor samples [31]. Regardless of the different specific designs, there is one common feature shared by most such screening studies. They all assume a monotone (either positive or negative) relationship between the end-point outcome and their genes of interest.

However, there remains the possibility that a true driver gene could actually exhibit a non-linear association with end-point observations. That is to say, both its up-regulation end and down-regulation can lead to aggressive tumor growth or metastasis, or vice versa. With a slight abuse of terms, "regulation" here includes any type of copy number variation, mutation, or RNA expression level change. Recently, Shen et al. explored the existence of such genes, which can potentially perform both oncogenic and tumor suppressive functions, through database searching and text mining [24]. They identified 83 genes that have dual functional annotation according to the literature. Most of these genes are transcription factors. They can both positively and negatively regulate transcription, which serves as the basis for their potential dual role in cancer development. These genes usually carry genomic mutation patterns similar to those of oncogenes, and expression patterns resembling those of tumor suppressor genes. TP53 is an example of one whose tumor suppressive effect, as exerted by activating DNA repair proteins, arresting the cell cycle and initiating apoptosis, is well known. On the other hand, more than $80 \%$ of the somatic and germline TP53 alterations found are missense mutations rather than nonsense or frame-shift mutations, which usually lead to loss of function. The strong selection to maintain expression of the full-length p53 mutant protein and its accumulation in the nucleus is an implication of gain-of-function and oncogenic mutation [26]. An in vivo knock in experiment has shown that many mutant p53 variants are essential for neoplastic transformation [29]. Another close example is Notch, which is an oncogene in cancer types like $\mathrm{T}$ cell acute lymphoblastic leukemia (ALL), and a tumor suppressor gene in other types like B cell ALL [18]. A more concrete example would be c-Myc whose dual role in leukemia was described by Uribesalgo et al. [30]. They showed that the c-Myc/RAR $\alpha$ complex could function either as an activator or a repressor based on the c-Myc phosphorylation status.
Although to the extent of our knowledge at present, there is no solid evidence of a gene that can perform both oncogenic and tumor suppressive effects in one cell line, the possibility cannot be ruled out. Such genes may be overlooked by traditional approaches, as these assume a linear association. Even if not a true bifunctional gene, a gene bearing a true function and a passenger event (e.g. a tumor suppressor gene coincidentally amplified with a nearby oncogene) can easily confound analysis, leading to its failure to be discovered as a hit. Therefore, it is important and worthwhile to explore whether there exists a non-linear association between genomic features and end-point outcomes, what the abundance is, and how it occurs if it does exist. As far as we know, no such study has been proposed to answer these questions.

In this study, we carried out a large-scale bioinformatics screen with the motivation to search for genes that have tri-modal association with end-point observations. First, we divided patients or cell lines into "lower than normal" ("low"), "similar to normal" ("middle") and "higher than normal" ("high") groups based on the expression levels of each investigated gene in tumor samples with respect to normal samples. To do this, we devised an algorithm based on Expectation-Maximization (EM) [9] that takes into consideration the expression levels of both normal samples and tumor samples for each gene. Then we focused on a specific scenario where candidate targets whose "low" and "high" groups of patients were both associated with worse survival and higher tumor grade compared to the "middle" group of patients. We termed this a "tri-modal" association.

This study will mainly focus on breast cancer, which is the most common type of invasive cancer in women. Breast tumors can be graded with the Nottingham Histologic Score system [25]. In this system, a grade of 1,2 or 3 is given to a breast tumor, where 3 has the poorest chance of prognostic survival. A number of tumor driver genes have been previously identified in breast cancers. For example, ERBB2, ESR1 and c-myc are breast tumor oncogenes; p53, p27, Skp2, BRCA-1 and BRCA-2 are breast tumor suppressors [20, 32]. Breast cancer can be divided into 5 subtypes according to the PAM50 assay [21], which include luminal A, luminal B, HER2-enriched, basal-like, and normal-like subtypes. The basal-like breast tumor subtype largely overlaps the triple negative type of breast cancer, which lacks or shows a low level of ESR1 and PGR expressions, and lacks ERBB2 amplification. Estrogen-receptor (ER) negative breast cancer, which generally includes basal and HER2 subtypes, is characterized by aggressive clinical behavior and resistance to hormone deprivation therapy [28]. In our study, we replicated our analysis across an array of breast tumor patient cohorts, including the following: (1) the Metabric study [8], where a 
total of $\sim 2000$ patients are available and divided into a discovery set and a validation set; (2) the Cancer Genome Atlas (TCGA) [5] breast cancer study, where 1000 patients are available; (3) the GSE18229 study [22], where 337 breast cancer patients are available; (4) the GSE20624 study [1], where 344 breast cancer patients are available; (5) the GSE20685 study [14], where 327 breast cancer patients are available; and (6) the GSE22133 study [12, 13], where 359 breast cancer patients are available.

\section{Results}

\section{Grouping of patients into 3 modes by EM algorithm}

We focused on the cases where the tumor patients can be grouped into "low", "middle" and "high" groups according to expression of a certain gene. The "middle" group should have expression levels similar to normal patients, while both "low" and "high" groups should have worse survival and higher tumor grades than "middle" group patients. This scenario enables a natural explanation that the "low" and "high" groups of patients suffer from a cancerous condition that deviated from the "middle" and normal patients, and the expression of this gene may be the cause for this cancerous condition. We devised an EM algorithm for this task. To test that the EM algorithm was working properly, we simulated the tumor population as a mixture of Gaussian $(-4,1)$, Gaussian $(0,1)$ and Gaussian $(3,1)$ with numbers of samples equal to 100,250 and 150 . We also simulated the normal population as Gaussian $(0,1)$ with number of samples equal to 50. The EM algorithm detected the mean vector to be $(-3.92,-0.076,2.93)$, mixing proportion to be $(0.21,0.59,0.29)$ and the standard deviation to be 1.006 , which are very close to the true parameters (Fig. 1a). We used the Metabric data as our primary dataset, where we perform the EM algorithm on discovery set against the normal set, and the validation set against the normal set, respectively. For example, Fig. 1b shows the distribution of the expression values for the gene ORMDL3 in the discovery set. The distribution of ORMDL3 in the validation set was very similar (Additional file 1: Figure S1). This screen was conducted on all 25235 genes available in the expression data and returned 6703 and 8706 genes with tri-modal distribution in the discovery set and validation set, respectively. The degree of trimodality varies greatly from weak to strong for these genes. In Fig. 1c, we showed the overlap between these two lists of genes. We also performed the trimodality search on the TCGA BRCA breast cancer patients. Figure 1c also shows the overlap between the common trimodal genes found in the Metabric dataset and the trimodal genes found in the TCGA dataset, comparing only genes that were available
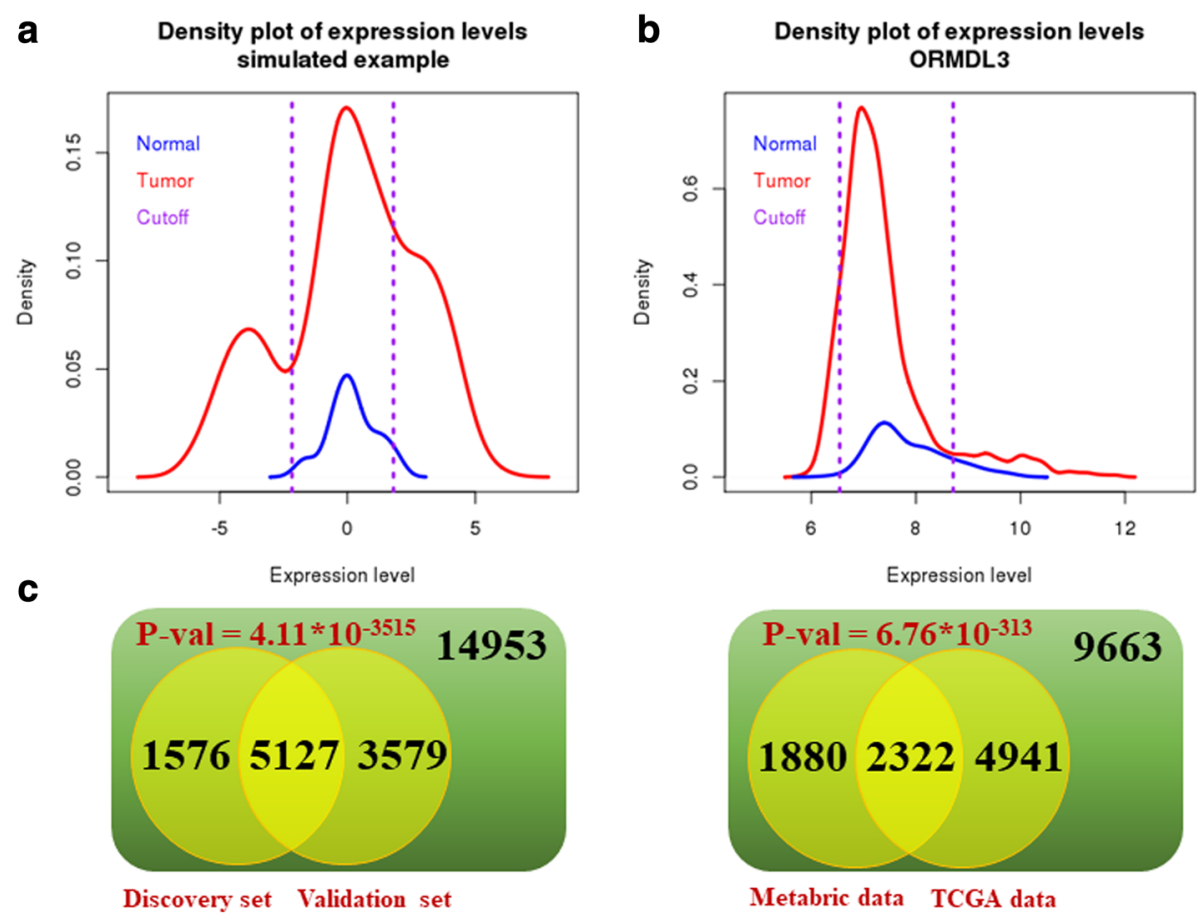

Fig. 1 Applied EM algorithm to discover trimodal genes. a A simulated example to verify the validity of the EM algorithm. b The distribution of the expression values for the gene ORMDL3 in the Metabric discovery set. c The common genes found to have trimodal distribution between the Metabric discovery set vs. Metabric validation set, and between the Metabric data and TCGA data. Hypergeometric $p$ is given to show the significance of overlap of trimodality or non-trimodality across different cohorts of patients 
in both datasets. The hypergeometric $p$ values show that genes tended to consistently show trimodality or non-trimodality across different cohorts of patients.

\section{Identify genes with tri-modal association with prognostic survival and tumor grade}

Using each gene that had a trimodal distribution and each mode whose proportion was at least $5 \%$ within both the Metabric discovery set and Metabric validation set, we tried to investigate whether both the "high" and "low" mode correlated significantly $(p<0.05)$ with worse prognostic survival and higher tumor grade than the "middle" mode. No gene satisfies this criterion, but one gene, ORMDL3, was very close (Fig. 2a and Table 1). The EM algorithm detected 10.0 and $7.7 \%$ of all discovery set patients to be in the "low" and "high" modes; and 10.0 and $9.9 \%$ of all validation set patients to be in the "low" and "high" modes. To test if this observation was robust, we tried to replicate the analysis in the TCGA BRCA cohort and 4 smaller cohorts, including GSE18229, GSE20624, GSE20685, and GSE22133. In these four smaller cohorts, there were no normal patients to conduct the EM algorithm. Therefore, we took the average of the proportions found in the Metabric cohorts and split each cohort into 10.0, 81.1 and 8.8\% according to the expression levels of ORMDL3. Figure $2 b$ shows the results of the survival analysis. It can be seen that the trimodal association between ORMDL3 and prognostic survival was significant (p12 $<0.05$ and p $23<0.05)$ for GSE20624. This relationship was non-significant for GSE18229, GSE20685 and GSE22133, but at least the trimodal trend was correct (p12<0.5 and p $23<0.5$ ). Table 1 shows the association between ORMDL3 expression and tumor grade. It can be seen that patients whose ORMDL3 expression fell into the low mode always had a significantly $(p<0.05)$ higher grade than those whose ORMDL3 expression fell into the middle mode. Patients whose ORMDL3 expression fell into the high mode didn't always have significantly $(p<0.05)$ higher grades than those whose ORMDL3 expression fell into the middle mode, but the trend was still correct $(p<0.5)$ in most cases.

\section{The phenotype of ORMDL3 amplification may be artefact of nearby ERBB2 expression}

Overall, we conclude that both the up-regulation and down-regulation of ORMDL3 were correlated with bad

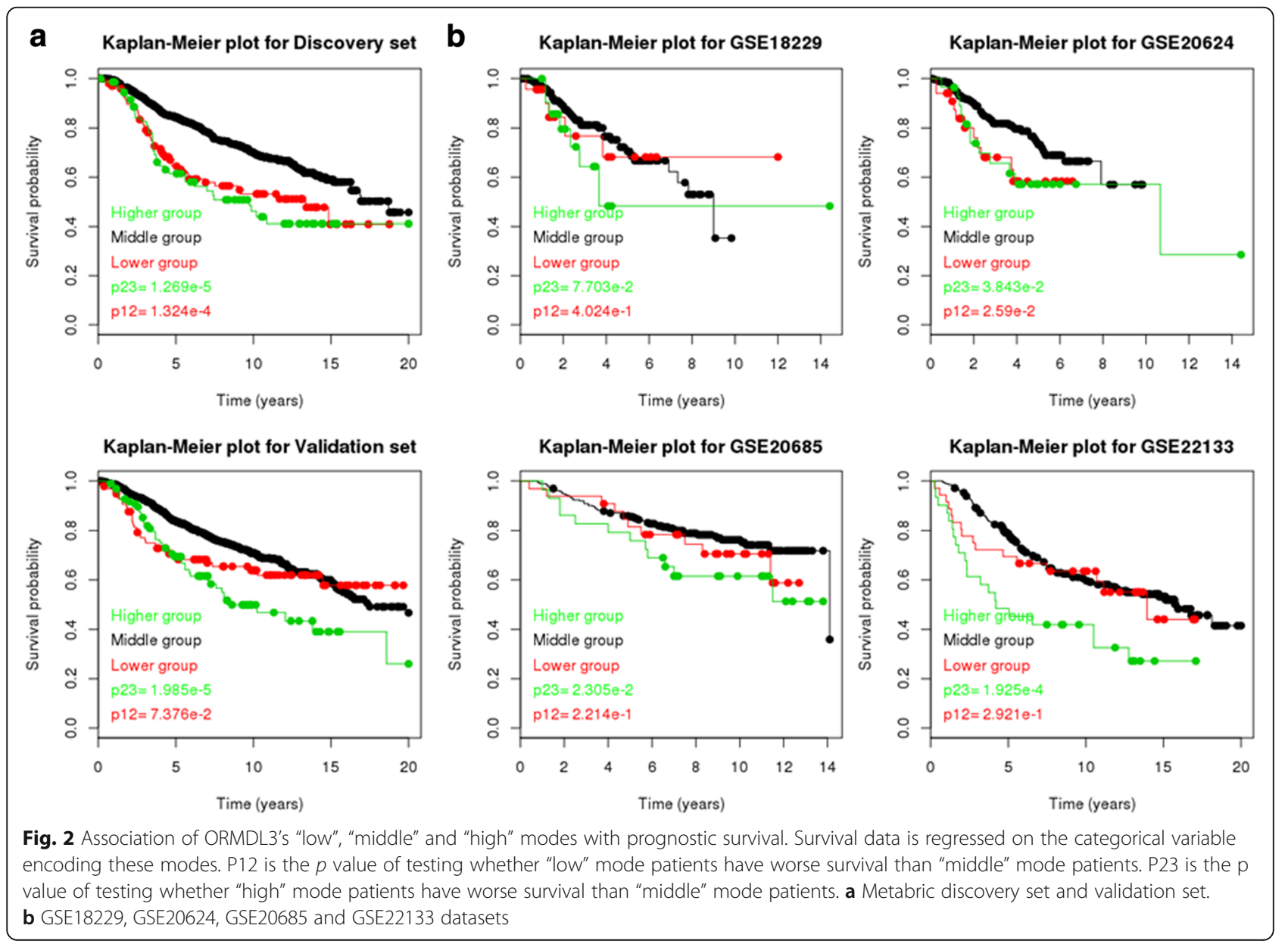


Table 1 Association of ORDML3 trimodal expression with tumor grade

\begin{tabular}{|c|c|c|c|c|c|c|c|c|}
\hline \multirow[t]{3}{*}{ Data set } & \multicolumn{6}{|c|}{ Patient number } & \multicolumn{2}{|l|}{$P$-value } \\
\hline & \multicolumn{3}{|c|}{ Expression } & \multicolumn{3}{|c|}{ Tumor grade } & \multirow[t]{2}{*}{ "Low" vs. "Middle" } & \multirow[t]{2}{*}{ "High" vs. "Middle" } \\
\hline & low & middle & high & Stage 1 & Stage 2 & Stage 3 & & \\
\hline Metabric discovery & 100 & 820 & 77 & 72 & 415 & 510 & $9.0 \times 10^{-7}$ & $4.6 \times 10^{-10}$ \\
\hline Metabric validation & 94 & 719 & 92 & 98 & 360 & 447 & $1.1 \times 10^{-5}$ & $2.9 \times 10^{-8}$ \\
\hline GSE18229 & 10 & 186 & 27 & 24 & 74 & 125 & $2.7 \times 10^{-3}$ & $3.9 \times 10^{-1}$ \\
\hline GSE20624 & 34 & 253 & 29 & 19 & 97 & 200 & $3.9 \times 10^{-2}$ & $7.8 \times 10^{-1}$ \\
\hline GSE22133 & 25 & 187 & 20 & 26 & 100 & 106 & $1.7 \times 10^{-3}$ & $5.4 \times 10^{-2}$ \\
\hline
\end{tabular}

Tailed $\mathrm{p}$ value is for the null hypothesis that "low" ("high") group patients tend to have lower grade tumors when compared to "middle" group patients. GSE20685 does not have tumor grade data, so the $p$ value is not calculated

prognosis and higher tumor grade in breast cancer patients, although this observation did not reach statistical significance in some small validation datasets. We then asked whether ORMDL3 was the driving factor for both the up-regulation phenotype and down-regulation phenotype. We noticed that ORMDL3 is only about 200 kb away from ERBB2/HER2 (Fig. 3a), which is a well-known tumor driver in multiple cancers, including breast cancer [11]. $15-25 \%$ of breast tumors carry a high-level amplification of ERBB2 [10], and ERBB2-overexpressing in breast cancer leads to substantially lower overall survival rates [27].

We hypothesized that the phenotype of up-regulation of ORMDL3 is a passenger event of nearby ERBB2's

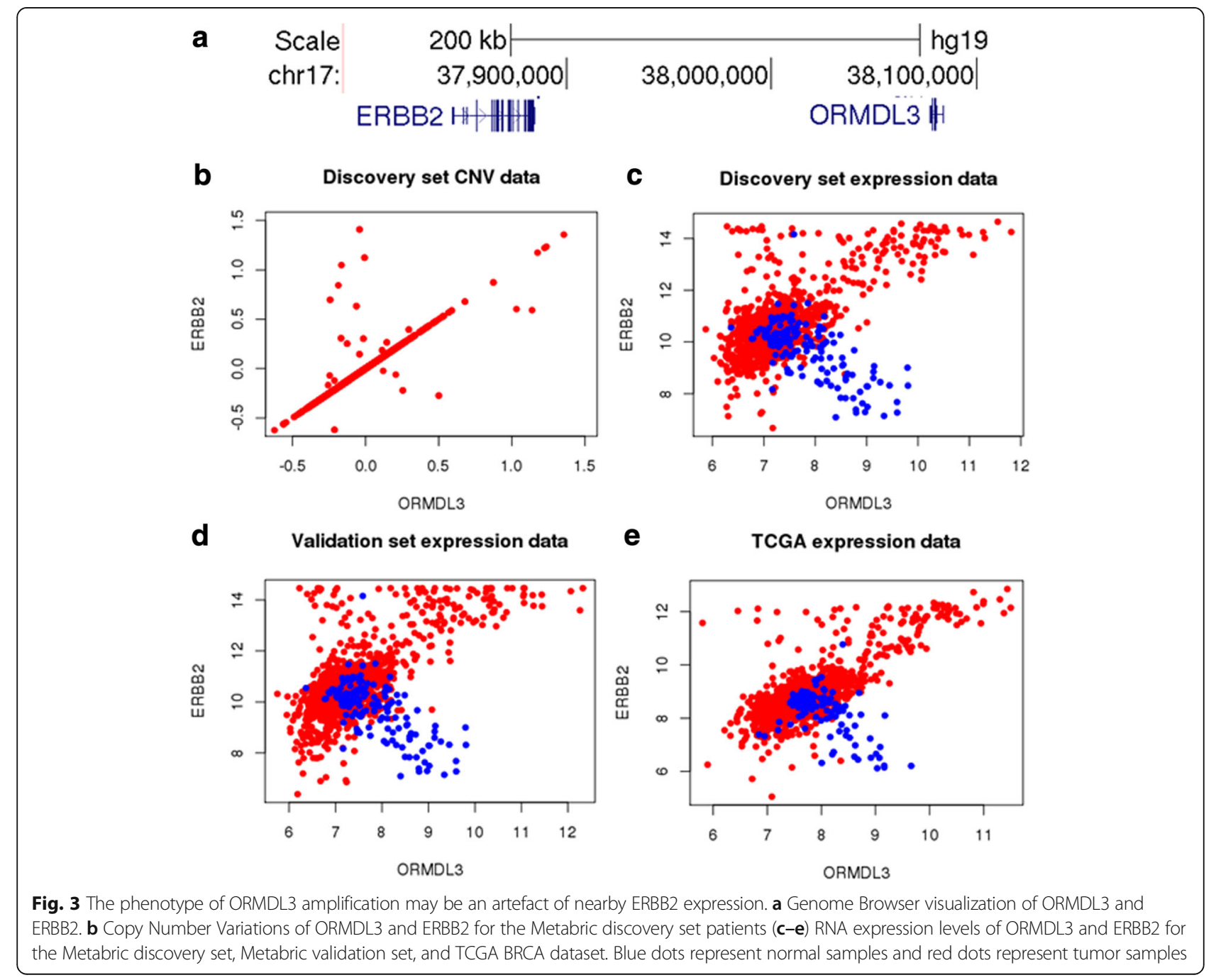


amplification. Indeed, when we plotted the Copy Number Variations of ORMDL3 and ERBB2 for the Metabric discovery set patients in Fig. 3b, we could see that ORMDL3 and ERBB2 were often amplified or deleted together. When ORMDL3 was amplified, ERBB2 was always amplified, but not vice versa. This could be replicated in the Metabric validation dataset and TCGA BRCA dataset (Additional file 1: Figure S2). Consistent with CNV data, the ORMDL3 and ERBB2 expression levels were positively correlated for the tumor samples, but with a significant portion of outliers in the upper-left corner (Fig. 3c-e). Interestingly, in normal samples, ORMDL3 and ERBB2 were negatively correlated in all three datasets examined. In addition, tumor and normal samples tended to occupy different regions in the ORMDL3-by-ERBB2 graphs.

Moreover, we calculated the relationship between gene essentiality vs. gene expression. For ORMDL3 (Additional file 1: Figure S3a), expression has a slightly positive association with gene essentiality. But for an oncogene, the higher it is expressed, the more likely the tumor cell line is reliant on this gene's expression for survival. In turn, this cell line is more sensitive to knockdown of the oncogene, leading to a more negative gene essentiality score. Indeed, the expression-by-essentiality plots show strong negative associations for some oncogenes (Additional file 1: Figure S3b-e), but not for tumor suppressors (Additional file 1: Figure S3f-k) [6, 16]. Although inconclusive, this analysis suggests that ORMDL3 has no oncogenic effect.

\section{ORMDL3 may be a breast tumor suppressor}

Based on the above-mentioned evidence, it is reasonable to suspect that the up-regulation of ORMDL3 is merely a passenger event of ERBB2 amplification. However, we hypothesized that the association between down-regulation of ORMDL3 and worse survival prognosis as well as higher tumor grade is due to the possible tumor suppressor effect of ORMDL3. To investigate this hypothesis, we conducted a multivariable analysis incorporating the 3 modes of ORMDL3 expression together with other variables for the Metabric discovery set survival data (Table 2). These variables include the expression level of ERBB2 as well as many other clinical variables. According to the table, the association of the up-regulation of ORMDL3 with worse survival is no longer significant $(p=0.72)$, while the down-regulation of ORMDL3 with worse survival is still significant $(p=0.002)$ after adjustment. We also extended this analysis to the other datasets, though not all of them fully captured these biological and clinical variables. So in this analysis, we conducted multivariable regression of the 3 modes of ORMDL3 expression only with ERBB2 for both survival and tumor grade data (Additional file 1: Table S1). We can see that the $p$ values representing the down-regulation of ORMDL3 did not
Table 2 Multivariable survival analysis with ORMDL trimodal expression and other variables

\begin{tabular}{lll}
\hline Variables & coefficient & p-value \\
\hline ORMDL expression ("low" vs. "middle") & 0.513 & 0.002 \\
ORMDL expression ("high" vs. "middle") & -0.140 & 0.72 \\
ERBB2 expression & 0.182 & 0.001 \\
ESR1 expression & -0.063 & 0.87 \\
PGR expression & -0.175 & 0.99 \\
Pam50subtype - Her2 & -0.206 & 0.78 \\
Pam50subtype - LumAB & -0.273 & 0.81 \\
Pam50subtype - Normal & 0.077 & 0.40 \\
Age at diagnosis & 0.148 & 0.002 \\
Stage & 0.024 & 0.36 \\
Lymph nodes positive & 0.110 & $<0.001$ \\
\hline
\end{tabular}

Analysis was done in Metabric discovery set

change too much from the univariate $\mathrm{p}$ values, while $\mathrm{p}$ values representing the up-regulation of ORMDL3 are mostly much less significant than the univariate $\mathrm{p}$ values. These results again confirmed our speculation that up-regulation of ORMDL3 is an artefact while ORMDL3 may be a new tumor suppressor.

\section{Discussion}

ORMDL3 is an endoplasmic reticulum-located transmembrane protein. It is mainly known as a negative regulator of sphingolipid synthesis [3], and it is involved in asthma as well as a series of autoimmune disorders [23]. However, currently few research papers have demonstrated whether it is involved in cancer. To validate its hypothetic role as a tumor suppressor, further experimental validation would need to be carried out. Similar analysis can also be carried out in the future in other cancer datasets to identify potential functional genes in cancer that may be missed by traditional studies.

\section{Conclusions}

In this study, we proposed an EM model to detect genes with trimodal expression in cancer patients to answer our specific question of interest: can a gene be both an oncogene and a tumor suppressor in a certain scenario? Applying our EM algorithm to the Metabric breast cancer dataset, we identified the gene ORMDL3, whose low and high expression are both associated with higher tumor grade and worse survival outcome. Down-stream analysis suggests the oncogenic effect of ORMDL3 may be an artefact by its nearby oncogene ERBB2 amplification, while its tumor suppressor role cannot be ruled out. Current research into ORMDL3 is focused on asthma and autoimmune diseases, so the functional study of its role in cancer is still blank. Future bench 
work is needed to validate its tumor suppressive effect in breast cancer. Taken together, this study provides a novel angle to look for oncogenes and tumor suppressors, linking trimodal gene abundance to endpoint observation.

\section{Methods}

\section{Curation of breast cancer studies}

The Metabric study datasets were downloaded from EMBL-EBI with the study ID EGAS00000000083. Study datasets were comprised of the discovery set and the validation set, as well as a third smaller group of normal control samples. For the expression data of each set of samples, probe-level data were aggregated to the gene level and each sample was adjusted using quantile normalization. For the copy number variation variant data, each gene's $\mathrm{CNV}$ status was found by calculating the mean of the values of the probes covering that gene. The TCGA Breast invasive carcinoma (BRCA) study data were also downloaded and contained mostly tumor samples and some normal samples. The HiSeq expression data were log transformed and median centered. The BRCA CNV data were downloaded from Firehose, and GISTIC gene-level output were used directly. For the GSE18229 study and the GSE20624 study, expression data were downloaded from the UNC microarray database, aggregated from the probe-level to the gene-level and quantile normalized. For the GSE20685 study, the expression data were downloaded from the GEO database. For the GSE22133 study, the expression data were aggregated from the probe level to the gene level and quantile normalized. For the CNV data, the values of the probes covering each gene were averaged to become the CNV status of that gene.

\section{EM algorithm}

We devised an EM algorithm to separate the whole tumor patient population into 3 groups, "higher than normal", "similar to normal" and "lower than normal". To do this, we assumed that the expression values of a certain gene in the tumor patient population were a mixture of 3 Gaussian distributions (3 modes), corresponding to each of the 3 groups mentioned above. We assumed those of the normal patient corresponded only to the middle component. To avoid assignment of a patient to an unreasonable mode, we assumed these 3 Gaussian distribution shared the same variance. Then the log likelihood function could be written as:

$$
\begin{aligned}
L L & \left(\overrightarrow{\mathrm{x}}_{\text {tumor }}, \overrightarrow{\mathrm{x}}_{\text {normal }} ; \vec{\pi}, \vec{\mu}, \sigma\right) \\
& =\sum_{i=1}^{\text {\#tumor }} \log \left(\sum_{j=1}^{3} f\left(\mathrm{x}_{\text {tumor }, i} ; \mu_{j}, \sigma\right) \times \pi_{j}\right) \\
& +\sum_{i=1}^{\# \text { normal }} \log \left(f\left(\mathrm{x}_{\text {normal }, i} ; \mu_{2}, \sigma\right)\right)
\end{aligned}
$$

$f(x ; \mu, \sigma)=\frac{1}{\sqrt{2 \pi} \sigma} e^{-\frac{(x-\mu)^{2}}{2 \sigma^{2}}}$ is the density function of normal distribution. $\vec{x}_{\text {tumor }}$ and $\vec{x}_{\text {normal }}$ are the vectors of expression levels of a certain gene in the tumor patient population and normal patient population. $\vec{\pi}$ is a 3 -element vector specifying the proportion of patients that belong to each of the 3 modes. $\vec{\mu}$ is a 3-element vector specifying the mean of the 3 Gaussian distributions, subject to $\mu_{1} \leq \mu_{2}, \mu_{2} \leq \mu_{3}$. $\sigma$ is the standard deviation of the 3 Gaussian distributions.

For each round, the EM algorithm was started by updating the responsibilities $\vec{\gamma}$, which is a vector with

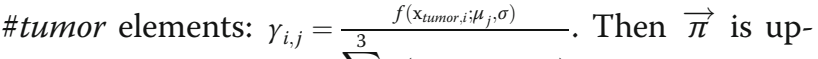

$$
\sum_{k=1}^{3} f\left(\mathrm{x}_{\text {tumor }, i} ; \mu_{k}, \sigma\right)
$$

dated by $\pi_{j}=\frac{\sum_{i=1}^{\# \text { tumor }} \gamma_{i, j}}{\# \text { tumor }}, \vec{\mu}$ is updated by $\mu_{j}=$ $\frac{\sum_{i=1}^{\text {\#tumor }} x_{\text {tumor }, i} \gamma_{i, j}+I(j=2) \sum_{i=1}^{\text {\#normal }} x_{\text {normal }, i}}{\sum_{i=1}^{\text {\#tumor }} \gamma_{i, j}+I(j=2) \times \text { \#normal }}(j=1,2,3)$, but the inequality bounds require that:

$$
\text { if } \quad \mu_{1}>\mu_{2}, \quad \mu_{2} \leq \mu_{3}, \quad \text { then } \quad \mu_{1}=\mu_{2}=
$$

$$
\frac{\sum_{j=1}^{2} \sum_{i=1}^{\# \text { tumor }} x_{\text {tumor }, i} \gamma_{i, j}+I(j=2) \sum_{i=1}^{\# \text { normal }} x_{\text {normal }, i}}{\sum_{j=1}^{2} \sum_{i=1}^{\# \text { tumor }} \gamma_{i, j}+I(j=2) \times \text { \#normal }} ;
$$

$$
\text { if } \quad \mu_{1} \leq \mu_{2}, \quad \mu_{2}>\mu_{3}, \quad \text { then } \quad \mu_{2}=\mu_{3}=
$$

$$
\frac{\sum_{j=2}^{3} \sum_{i=1}^{\# \text { tumor }} x_{\text {tumor }, i} \gamma_{i, j}+I(j=2) \sum_{i=1}^{\# \text { normal }} x_{\text {normal }, i}}{\sum_{j=2}^{3} \sum_{i=1}^{\# \text { tumor }} \gamma_{i, j}+I(j=2) \times \# \text { normal }} ;
$$$$
\text { and if } \quad \mu_{1}>\mu_{2}, \quad \mu_{2}>\mu_{3}, \quad \text { then }
$$$$
\mu_{1}=\mu_{2}=\mu_{3}=\frac{\sum_{j=1}^{3} \sum_{i=1}^{\# \text { \#umor }} x_{\text {tumor }, i} \gamma_{i, j}+I(j=2) \sum_{i=1}^{\# \text { \#ormal }} x_{\text {normal }, i}}{\sum_{j=1}^{3} \sum_{i=1}^{\# \text { tumor }} \gamma_{i, j}+I(j=2) \times \text { \#normal }} .
$$

$$
\left[\frac{\left.\sum_{i=1}^{\text {Finally, } \sum_{j=1}^{3} \gamma_{i, j}\left(\mathrm{x}_{\text {tumor }, i}-\mu_{j}\right)^{2}+\sum_{i=1}^{\text {\#normal }}\left(\mathrm{x}_{\text {normal }, i}-\mu_{2}\right)^{2}}\right]}{\sum_{i=1}^{\text {\#tumor }} \sum_{j=1}^{3} \gamma_{i, j}+\text { \#normal }}\right] .
$$


The EM iterations were stopped when the log likelihood reached convergence. When $\mu_{1}<\mu_{2}, \mu_{2}<\mu_{3}$, and $\pi_{i}>0.01$, $i=1,2,3$ were all satisfied, this gene was said to exhibit trimodality distribution. Then two cutoff values were calculated by cutoff $_{12}=\frac{\mu_{1}^{2}-\mu_{2}^{2}-2 \sigma^{2} \log \left(\frac{\pi_{1}}{\pi_{2}}\right)}{2\left(\mu_{1}-\mu_{2}\right)}$ and cutoff $_{23}$ $=\frac{\mu_{2}^{2}-\mu_{3}^{2}-2 \sigma^{2} \log \left(\frac{\pi_{2}}{\pi_{3}}\right)}{2\left(\mu_{2}-\mu_{3}\right)}$. Sometimes cutoff $_{12}>\mu_{2}$ or cutoff $_{12}<\mu_{1}$ could occur. When that happened, an ad hoc rule applied to set cutoff $_{12}$ at the $10 \%$ quantile of the expression values of the tumor samples. Similarly, cutoff ${ }_{23}$ was set at the $90 \%$ quantile when cutoff $_{23}>\mu_{3}$ or cutoff $_{23}<\mu_{2}$. Finally the true membership of each tumor sample to the three modes was decided by comparing their expression values to cutoff $_{12}$ and cutoff $_{23}$. An empirical $\pi$ was calculated by the proportion of tumor patients belonging to each mode.

\section{Gene essentiality analysis}

The gene essentiality screening data were downloaded from the 2012 Cancer Discovery study [19]. In this study, a continuous GARP score was defined for each gene in every cell line. A lower score for a gene meant that the cell line was more reliant on the expression of this gene for survival. We used the expression data downloaded from the Cancer Cell Encyclopedia (CCLE) website [2]. The whole CCLE dataset contained the expression data of 58 breast cancer cell lines. 29 of these cell lines were also used in the gene essentiality screening study.

\section{Statistical tests}

Survival analysis performed in this study was done using functions from the $\mathrm{R}$ survival package. To test the tri-modal association of each gene's expression level with overall survival, the "low", "middle", and "high" categorical variables were input into the Cox proportional hazard model, with or without adjusting for other variables. The $P$ value for the "low" group was assigned by testing the null hypothesis that "low" group patients had no worse overall survival than "middle" group patients, and the same applied for "high" group $p$ values. All survival analysis was censored at 20 years.

To test the proportional trend of two groups of patients in tumor graded 1, 2 and 3, a modified version of prop.trend.test function from the stats $\mathrm{R}$ package was used. The $\mathrm{p}$ value generated by prop.trend.test was from a two-tailed test, while a one-tailed $\mathrm{p}$ value was calculated from it by examining the sign of the coefficient. The one-tailed p value was for the null hypothesis that "low" ("high") group patients tended to have lower grade tumors when compared to "middle" group patients. To compare "low" vs. "middle" groups for example, the test in essence generated a smaller $\mathrm{p}$ value when more advanced grade tumors were more likely to be "low" group patients rather than "middle" group patients.

\section{Additional file}

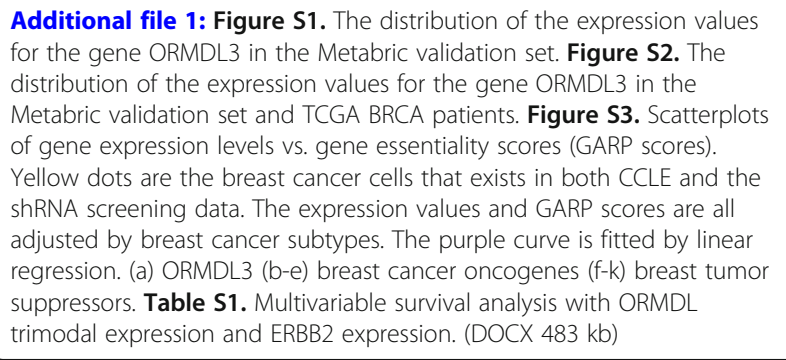

\section{Abbreviations}

ALL: Acute lymphoblastic leukemia; CCLE: Cancer Cell Encyclopedia; CCLE: Copy number variations; EM: Expectation-Maximization; RNAi: RNA interference; shRNA: Small hairpin RNA; SNP: Single nucleotide polymorphism

\section{Acknowledgements}

We thank Jessie Norris for language editing of the manuscript, and the anonymous reviewers for their valuable advice on this paper.

\section{Funding}

This study was supported by the National Institutes of Health (NIH) [R01GM115473, R01HL087564, R03ES026397, and 1P50CA19651601and the Cancer Prevention and Research Institute of Texas [CPRIT RP180805]. The funders had no role in the design of the study and collection, analysis, and interpretation of data and in writing the manuscript.

\section{Availability of data and materials}

The Metabric breast cancer dataset used in the study can be downloaded at https://www.ebi.ac.uk/ega/studies/EGAS00000000083. The TCGA BRCA dataset can be found at https://portal.gdc.cancer.gov/projects/TCGA-BRCA. The GEO datasets are available in the GEO database with accession numbers GSE18229, GSE20624, GSE20685 and GSE22133. The GARP score used in the study is included in Marcotte et al. [19]. The CCLE dataset can be downloaded from https://portals.broadinstitute.org/ccle. The source code of the EM algorithm and all the analysis is available at https://github.com/ Minzhe/trimodal.

\section{Authors' contributions}

YX and TW decided the direction of research and drove the project. MZ and TW drafted the manuscript. YX revised the manuscript. TW formulated the proposed model. MZ implemented the method, preprocessed the data and conducted the major analysis. RS and PS provided preliminary lab validation of the finding. All authors read and approved the final manuscript.

Ethics approval and consent to participate Not applicable.

\section{Consent for publication \\ Not applicable.}

\section{Competing interests}

The authors declare that they have no competing interests.

\section{Publisher's Note}

Springer Nature remains neutral with regard to jurisdictional claims in published maps and institutional affiliations.

\section{Author details}

${ }^{1}$ Department of Clinical Sciences, Quantitative Biomedical Research Center, University of Texas Southwestern Medical Center, 5323 Harry Hines Blvd, Dallas, TX 75390, USA. ${ }^{2}$ Harold C. Simmons Comprehensive Cancer Center, University of Texas Southwestern Medical Center, 5323 Harry Hines Blvd, Dallas, TX 75390, USA. ${ }^{3}$ Center for the Genetics of Host Defense, University of Texas Southwestern Medical Center, 5323 Harry Hines Blvd, Dallas, TX 75390, 
USA. ${ }^{4}$ Department of Pediatrics, Division of Pulmonary and Vascular Biology, University of Texas Southwestern Medical Center, 5323 Harry Hines Blvd, Dallas, TX 75390, USA. ${ }^{5}$ Department of Bioinformatics, University of Texas Southwestern Medical Center, 5323 Harry Hines Blvd, Dallas, TX 75390, USA.

Received: 21 August 2018 Accepted: 11 December 2018

Published online: 08 January 2019

\section{References}

1. Anders $\mathrm{CK}$, et al. Breast carcinomas arising at a young age: unique biology or a surrogate for aggressive intrinsic subtypes? I Clin Oncol Off J Am Soc Clin Oncol. 2011;29:e18-20.

2. Barretina J, et al. The Cancer cell line encyclopedia enables predictive modelling of anticancer drug sensitivity. Nature. 2012;483:603-7.

3. Breslow DK, et al. Orm family proteins mediate sphingolipid homeostasis. Nature. 2010;463:1048-53.

4. Bric $\mathrm{A}$, et al. Functional identification of tumor-suppressor genes through an in vivo RNA interference screen in a mouse lymphoma model. Cancer Cell. 2009;16:324-35.

5. Cancer Genome Atlas, N. Comprehensive molecular portraits of human breast tumours. Nature. 2012;490:61-70.

6. Cipriano R, et al. FAM83B mediates EGFR- and RAS-driven oncogenic transformation. J Clin Invest. 2012;122:3197-210.

7. Croce CM. Oncogenes and cancer. N Engl J Med. 2008;358:502-11.

8. Curtis C, et al. The genomic and transcriptomic architecture of 2,000 breast tumours reveals novel subgroups. Nature. 2012:486:346-52.

9. Dempster AP, et al. Maximum likelihood from incomplete data via the EM algorithm. J R Stat Soc. 1977;39:1-38.

10. Haverty PM, et al. High-resolution genomic and expression analyses of copy number alterations in breast tumors. Genes Chromosomes Cancer. 2008;47: 530-42.

11. Herter-Sprie GS, et al. Activating mutations in ERBB2 and their impact on diagnostics and treatment. Front Oncol. 2013;3:86.

12. Holm K, et al. Characterisation of amplification patterns and target genes at chromosome 11q13 in CCND1-amplified sporadic and familial breast tumours. Breast Cancer Res Treat. 2012;133:583-94.

13. Jonsson $\mathrm{G}$, et al. Genomic subtypes of breast cancer identified by arraycomparative genomic hybridization display distinct molecular and clinical characteristics. Breast Cancer Res : BCR. 2010;12:R42.

14. Kao KJ, et al. Correlation of microarray-based breast cancer molecular subtypes and clinical outcomes: implications for treatment optimization. BMC Cancer. 2011;11:143.

15. Koso $\mathrm{H}$, et al. Identification of FoxR2 as an oncogene in medulloblastoma. Cancer Res. 2014;74:2351-61.

16. Lee EY, Muller WJ. Oncogenes and tumor suppressor genes. Cold Spring Harb Perspect Biol. 2010;2:a003236.

17. Levine AJ. The tumor suppressor genes. Annu Rev Biochem. 1993;62:623-51.

18. Lobry $C$, et al. Oncogenic and tumor suppressor functions of Notch in cancer: it's NOTCH what you think. J Exp Med. 2011;208:1931-5.

19. Marcotte R, et al. Essential gene profiles in breast, pancreatic, and ovarian cancer cells. Cancer Discov. 2012;2:172-89.

20. Osborne C, et al. Oncogenes and tumor suppressor genes in breast cancer: potential diagnostic and therapeutic applications. Oncologist. 2004;9:361-77.

21. Parker JS, et al. Supervised risk predictor of breast cancer based on intrinsic subtypes. J of Clin Oncol : official journal of the American Society of Clinical Oncology. 2009;27:1160-7.

22. Prat A, et al. Phenotypic and molecular characterization of the claudin-low intrinsic subtype of breast cancer. Breast Cancer Res : BCR. 2010;12:R68.

23. Qiu R, et al. Signal transducer and activator of transcription 6 directly regulates human ORMDL3 expression. FEBS J. 2013;280:2014-26.

24. Shen L, Shi Q, Wang W. Double agents: genes with both oncogenic and tumor-suppressor functions. Oncogenesis. 2018;7(3):25.

25. Simpson JF, et al. Prognostic value of histologic grade and proliferative activity in axillary node-positive breast cancer: results from the eastern cooperative oncology group companion study, EST 4189. J Clin Oncol : official journal of the American Society of Clinical Oncology. 2000;18:2059-69.

26. Soussi T, Wiman KG. TP53: an oncogene in disguise. Cell Death Differ. 2015; 22(8):1239.

27. Tan M, Yu D. Molecular mechanisms of erbB2-mediated breast cancer chemoresistance. Adv Exp Med Biol. 2007;608:119-29.
28. Tang $\mathrm{H}$, et al. Decreased BECN1 mRNA expression in human breast Cancer is associated with estrogen receptor-negative subtypes and poor prognosis. EBioMedicine. 2015;2:255-63.

29. Terzian T, Suh YA, Iwakuma T, Post SM, Neumann M, Lang GA, et al. The inherent instability of mutant p53 is alleviated by Mdm2 or p16INK4a loss. Genes Dev. 2008;22(10):1337-44.

30. Uribesalgo I, Benitah SA, Di Croce L. From oncogene to tumor suppressor: the dual role of Myc in leukemia. Cell Cycle. 2012;11(9):1757-64.

31. Wrzeszczynski $\mathrm{KO}$, et al. Identification of tumor suppressors and oncogenes from genomic and epigenetic features in ovarian cancer. PLoS One. 2011;6:e28503.

32. Yuan $Y$, et al. A sparse regulatory network of copy-number driven gene expression reveals putative breast cancer oncogenes. IEEE/ACM Trans Comput Biol Bioinform / IEEE, ACM. 2012;9:947-54.
Ready to submit your research? Choose BMC and benefit from:

- fast, convenient online submission

- thorough peer review by experienced researchers in your field

- rapid publication on acceptance

- support for research data, including large and complex data types

- gold Open Access which fosters wider collaboration and increased citations

- maximum visibility for your research: over $100 \mathrm{M}$ website views per year

At BMC, research is always in progress.

Learn more biomedcentral.com/submissions 